\title{
Targeting AML-associated FLT3 mutations with a type I kinase inhibitor
}

\author{
LaQuita M. Jones, ${ }^{1}$ Katelyn Melgar, ${ }^{2,3}$ Lyndsey Bolanos, ${ }^{2}$ Kathleen Hueneman, ${ }^{2}$ Morgan M. Walker, ${ }^{4}$ Jian-Kang Jiang, ${ }^{4}$ \\ Kelli M. Wilson, ${ }^{4}$ Xiaohu Zhang, ${ }^{4}$ Jian Shen, ${ }^{5}$ Fan Jiang, ${ }^{5}$ Patrick Sutter, ${ }^{4}$ Amy Wang, ${ }^{4}$ Xin Xu, ${ }^{4}$ Gregory J. Tawa, ${ }^{4}$ Scott B. Hoyt, ${ }^{4}$ \\ Mark Wunderlich, ${ }^{2}$ Eric O'Brien, ${ }^{1}$ John P. Perentesis, ${ }^{1}$ Daniel T. Starczynowski, ${ }^{2,6}$ and Craig J. Thomas ${ }^{4,7}$ \\ 'Division of Oncology and 'Division of Experimental Hematology and Cancer Biology, Cancer and Blood Diseases Institute, Cincinnati Children's Hospital Medical Center, Cincinnati, Ohio, USA. ${ }^{3}$ Immunology \\ Graduate Program, University of Cincinnati College of Medicine, Cincinnati, Ohio, USA. ${ }^{4}$ Division of Preclinical Innovation, National Center for Advancing Translational Sciences, NIH, Bethesda, Maryland, USA. \\ ${ }^{5}$ Viva Biotech Ltd., Shanghai, China. ${ }^{6}$ Department of Cancer Biology, University of Cincinnati College of Medicine, Cincinnati, Ohio, USA. ㄴ. Lymphoid Malignancies Branch, Center for Cancer Research, National \\ Cancer Institute (NCI), NIH, Bethesda, Maryland.
}

Tyrosine kinase domain (TKD) mutations contribute to acquired resistance to FMS-like tyrosine kinase 3 (FLT3) inhibitors used to treat FLT3-mutant acute myeloid leukemia (AML). We report a cocrystal structure of FLT3 with a type I inhibitor, NCCC1481, that retained potent binding and activity against FLT3 TKD and gatekeeper mutations. Relative to the current generation of advanced FLT3 inhibitors, NCCC1481 exhibited superior antileukemic activity against the common, clinically relevant FLT3-mutant AML cells in vitro and in vivo.

\section{Introduction}

Acute myeloid leukemia (AML) is associated with mutations in FMS-like tyrosine kinase 3 (FLT3) in approximately $30 \%$ of cases, including internal tandem duplications (ITDs) and point mutations in the tyrosine kinase domain (TKD) $(1,2)$. Given the prevalence of FLT3 mutations and their impact on disease progression, multiple FLT3 inhibitors (FLT3i) are now in various stages of clinical evaluation (3). Midostaurin, a multitargeted type I kinase inhibitor, was the first FDA-approved therapy for newly diagnosed FLT3-mutant AML (4). Quizartinib (AC220), a type II inhibitor with potent activity against FLT3-ITD, is currently being studied in phase III clinical trials, in combination with conventional chemotherapy, for newly diagnosed FLT3-ITD ${ }^{+}$AML (5). The type I inhibitor crenolanib is in phase III clinical trials for both newly diagnosed and relapsed/refractory FLT3-mutant AML. A secondgeneration FLT3 inhibitor, gilteritinib, exhibits potent activity against FLT3-ITD and several of the clinically relevant FLT3 mutants and was recently approved by the FDA (6). Although many patients with FLT3-ITD AML will initially respond to therapy, high rates of relapse and drug resistance contribute to a poor overall prognosis $(1,2)$. Various mechanisms of resistance to FLT3 inhibitors have been described, including upregulation of compensatory signaling pathways and mutations within the TKD of FLT3 (7-12). Although FLT3 TKD mutations have been described

Conflict of interest: MMW, JKJ, KM, DTS, and CJT are inventors of intellectual property involving NCGC1481 (WO 2018/038988 A2 [Compounds, Compositions, Methods for Treating Diseases, and Methods for Preparing Compounds]). MMW, JKJ, and CJT have assigned their rights to the NIH. DTS and KM have assigned their rights to the Cincinnati Children's Hospital Medical Center. DTS serves on the scientific advisory board of Kurome Therapeutics.

Copyright: @ 2020, American Society for Clinical Investigation.

Submitted: February 2, 2019; Accepted: January 8, 2020; Published: March 9, 2020.

Reference information: J Clin Invest. 2020;130(4):2017-2023.

https://doi.org/10.1172/JCI127907. in cases of newly diagnosed AML, they are known to be one of the primary mechanisms of acquired resistance to FLT3 inhibitor therapy. Two primary mutational sites following FLT3 inhibitor therapy have been shown to decrease the binding and inhibitory potential of many FLT3 inhibitors. These mutations involve residues in the activation loop (e.g., aspartate 835, D835) or the gatekeeper residue (i.e., phenylalanine 691, F691). Activation loop mutations have been described following exposure to AC220 and sorafenib, whereas F691L mutations have been found in patients following gilteritinib treatment (7-9). Mutations involving D835 are the most common genetic mechanism of relapse and resistance in FLT3-mutant AML following treatment with tyrosine kinase inhibitors $(7,11,12)$. Several studies demonstrated that type II inhibitors, such as AC220, lose potency in D835-mutant FLT3 AML (7, 11, 12). Structural consequences associated with mutations at the D835 site suggest that the equilibrium between the DFG-in (active form) and DFG-out (inactive form) of FLT3 is altered in ways detrimental to the binding competence of type II inhibitors $(13,14)$. Here, we report an ATP-binding site, type I inhibitor of FLT3 with potent biochemical activity against multiple, clinically relevant drug-resistant FLT3 mutations, excellent drug-like properties, and remarkable in vitro and in vivo activity in AML models, including those containing clinically relevant FLT3 TKD mutations.

\section{Results and Discussion}

Profiling of a series of small-molecule kinase inhibitors highlighted a previously reported series of 3-(pyridin-2-yl)imidazo [1,2-a]pyridines as potent inhibitors of FLT3 (15). We pursued a detailed evaluation of the structure activity relationships that govern this chemotype's activity versus FLT3 and versus FLT3-mutant AML. Optimization led to the identification of NCGC1481 (Figure $1 \mathrm{~A}$ ), which possessed strong biochemical potency compared with FLT3, acceptable pharmacokinetic properties (4.2 hours 
$t_{1 / 2} ; 3570 \mathrm{ng} / \mathrm{mL} \mathrm{C}_{\max } ; 0.083$ hours $\mathrm{T}_{\text {max }}$ [time that a drug reaches peak concentration in serum] at $30 \mathrm{mg} / \mathrm{kg}$ i.p.), and no observable hematologic toxicity in mice. NCGC1481 demonstrates 10-fold or greater selectivity versus more than $80 \%$ of tested kinases relative to FLT3 in biochemical assays using purified proteins and high kinase selectivity in situ (15). We next profiled NCGC1481 versus a commercial panel of known, clinically relevant mutations at the FLT3 TKD. NCGC1481 exhibited potent binding affinity for FLT3 and FLT3-ITD $\left(K_{D}=0.025\right.$ and $0.36 \mathrm{nM}$, respectively), common mutants including those at Asp 835 (D835X) $\left(K_{D}=0.01-0.29\right.$ $\mathrm{nM})$ and Phe 691 (F691L) $\left(K_{D}=0.012 \mathrm{nM}\right)$, as well as less common mutants including Lys $663(\mathrm{~K} 663 \mathrm{Q})\left(K_{D}=5.4 \mathrm{nM}\right)$, Asn $841(\mathrm{~N} 841)$ $\left(K_{D}=0.33 \mathrm{nM}\right)$, and $\operatorname{Arg} 834(\mathrm{R} 834)\left(K_{D}=0.3 \mathrm{nM}\right)$ (Table 1 and Supplemental Table 1; supplemental material available online with this article; https://doi.org/10.1172/JCI127907DS1). To provide context for these outcomes, we also profiled key drugs in active clinical development for FLT3-mutant AML (i.e., AC220, crenolanib, midostaurin, and giltertinib) as well approved drugs with known FLT3 and AML activity (ponatinib, cabozantinib, and sorafenib). These profiles demonstrated and/or confirmed that the prevalent D835 mutation was detrimental to the binding affinity of AC220, ponatinib, cabozantinib, and sorafenib (all type II inhibitors), whereas the binding affinity of crenolanib and gilteritinib (both type I inhibitors) was unaffected (Table 1 and Supplemental Table 1).

The correlative activity of NCGC1481 with crenolanib and gilteritinib strongly suggested that NCGC1481 was a type I inhibitor, binding to the DFG-in, active form of FLT3. To confirm a type I binding orientation for NCGC1481 versus FLT3, we pursued a cocrystal with a previously crystalized construct of FLT3. The resulting cocrystal structure did not resolve the pendant pyrrolidine ring of NCGC1481 but clearly defined the rest of the molecule including the hinge-binding imidazo[1,2-a] pyridine ring system. Critically, we confirmed that the binding conformation of NCGC1481 was that of a type I, DFG-in inhibitor (PDB: 6IL3) (Figure 1B). To further substantiate the structural consequences of the key FLT3 TKD mutations on both type I and type II inhibitors, we contrasted the structure of FLT3-bound NCGC1481 with FLT3-bound AC220 (Protein Data Bank [PDB]: 4XUF) (Figure 1B, right). Foremost, this side-by-side comparative view illustrates that Asp835 does not interact directly with either NCGC1481 or AC220. Rather, mutations at this site alter the binding affinity of type II inhibitors by corrupting the key hydrogen bond found between the Asp835 carboxylate and the Ser838 hydroxyl group, which helps stabilize the DFG-out, inactive conformation of FLT3 (Figure 1C, left). When mutated to residues (e.g., His, Val, Tyr) that produce a less energetically favorable H-bond (either through loss of an H-bond receptor or through suboptimal residue alignment) the equilibrium shifts toward the DFG-in, active state of the kinase. This is reflected by reports of hyperactivation of FLT3 signaling in D835-mutant populations $(7,11,12)$. Previously, Shah and colleagues reported a structure-based rationale for the loss of AC220 activity versus the F691L gatekeeper mutation highlighting an edge-to-face aromatic interaction with the phenylalanine residue (16). We note that the F691L gatekeeper mutation has significant consequences for the binding of other type II inhibitors (Supplemental Table 1). Our cocrystal highlights the fact that NCGC1481 is proximal to Phe 691 with a modest edge interaction (Figure 1C, right). The phenylalanine-to-leucine change does not, however, result in a loss of potency for NCGC1481 versus FLT3F691L. Mutations at other, less common sites (e.g., Lys 663, Asn 841, and Arg 834) had no noticeable interactions with NCGC1481 or effects on its binding affinity. These structural studies revealed the unique binding properties of NCGC1481 to FLT3, which predict activity against clinically relevant FLT3 mutations.

To examine whether the revealed binding affinity for NCGC1481 and the other key drugs profiled in this study altered their capacity to inhibit FLT3 signaling in leukemic cells, we studied isogenic AML cell lines harboring FLT3-ITD alone (MOLM14) and dual FLT3-ITD(D835Y) mutations [MOLM14(D835Y)], with D835Y representing the most common FLT3 TKD mutation. Consistent with its binding affinity for FLT3-ITD and clinically relevant mutants, NCGC1481 effectively suppressed FLT3-ITD and FLT3-ITD(D835Y) signaling in MOLM14 cells as demonstrated by reduced phosphorylation of FLT3 and STAT5 (Figure 1D). In contrast, AC220, midostaurin, and gilteritinib exhibited inhibition of FLT3 signaling in parental FLT3-ITD MOLM14 cells, but the activity of these agents in FLT3-ITD(D835Y) MOLM14 cells was compromised (Figure 1E). Crenolanib did not demonstrate potent inhibition of FLT3 in either the parental FLT3-ITD MOLM14 cells or the FLT3-ITD(D835Y) MOLM14 cells (Figure 1, D and E). These relative activities cannot be wholly explained by the binding affinity shown in Table 1 and suggest, unsurprisingly, that the cellular

Table 1. Dissociation constant of NCGC1481 and current generation of FLT3i versus FLT3 and key FLT3 mutants

\begin{tabular}{|c|c|c|c|c|c|}
\hline Key target & NCGC1481 & AC220 & Crenolamib & Midostaurin & Gilteritinib \\
\hline FLT3 & $0.025 \mathrm{nM}$ & $1.4 \mathrm{nM}$ & $0.2 \mathrm{nM}$ & $4 \mathrm{nM}$ & $1.4 \mathrm{nM}$ \\
\hline FLT3(D835H) & $0.27 \mathrm{nM}$ & $2.1 \mathrm{nM}$ & $0.2 \mathrm{nM}$ & $2.2 \mathrm{nM}$ & $0.7 \mathrm{nM}$ \\
\hline FLT3(D835V) & $0.01 \mathrm{nM}$ & $4.2 \mathrm{nM}$ & $0.016 \mathrm{nM}$ & $3.6 \mathrm{nM}$ & $0.16 \mathrm{nM}$ \\
\hline FLT3 (ITD) & $0.36 \mathrm{nM}$ & $7 \mathrm{nM}$ & $0.26 \mathrm{nM}$ & $4.1 \mathrm{nM}$ & $0.93 \mathrm{nM}$ \\
\hline FLT3 (ITD, D835V) & $0.01 \mathrm{nM}$ & $480 \mathrm{nM}$ & $0.23 \mathrm{nM}$ & $3.4 \mathrm{nM}$ & $0.73 \mathrm{nM}$ \\
\hline FLT3 (N841I) & $0.33 \mathrm{nM}$ & $1.5 \mathrm{nM}$ & $0.39 \mathrm{nM}$ & $2.3 \mathrm{nM}$ & $2.1 \mathrm{nM}$ \\
\hline FLT3 (R834Q) & $0.3 \mathrm{nM}$ & $12 \mathrm{nM}$ & $1.3 \mathrm{nM}$ & $27 \mathrm{nM}$ & $19 \mathrm{nM}$ \\
\hline FLT3 autoinhibition & $0.96 \mathrm{nM}$ & $580 \mathrm{nM}$ & $17 \mathrm{nM}$ & $120 \mathrm{nM}$ & $12 \mathrm{nM}$ \\
\hline
\end{tabular}


A<smiles>COc1cc2ncc(-c3cccc(NC4CCNC4)n3)n2cc1-c1cnn(C)c1</smiles>

NCGC1481
B

NCGC1481 bound to the DFG-in conformation of FLT3

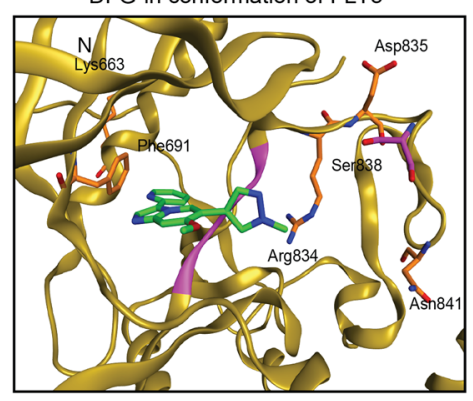

AC220 bound to the DFG-out conformation of FLT3

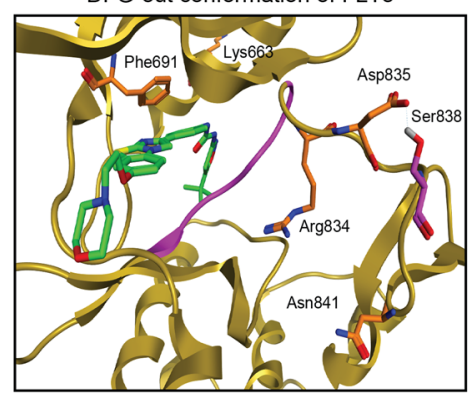

C
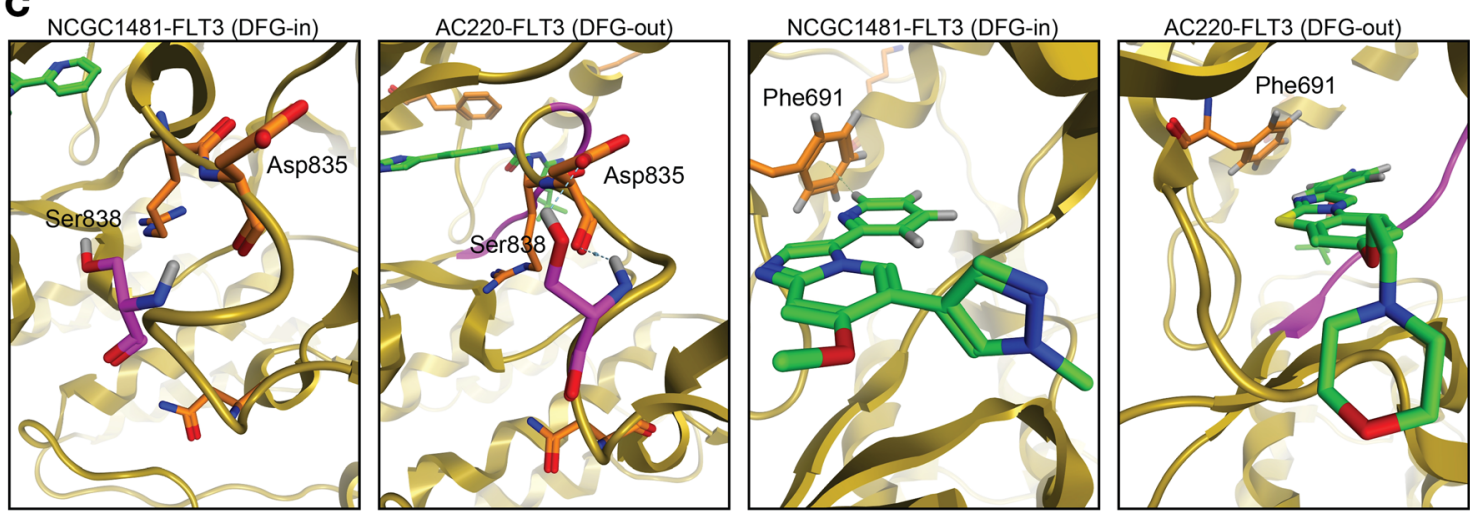

D
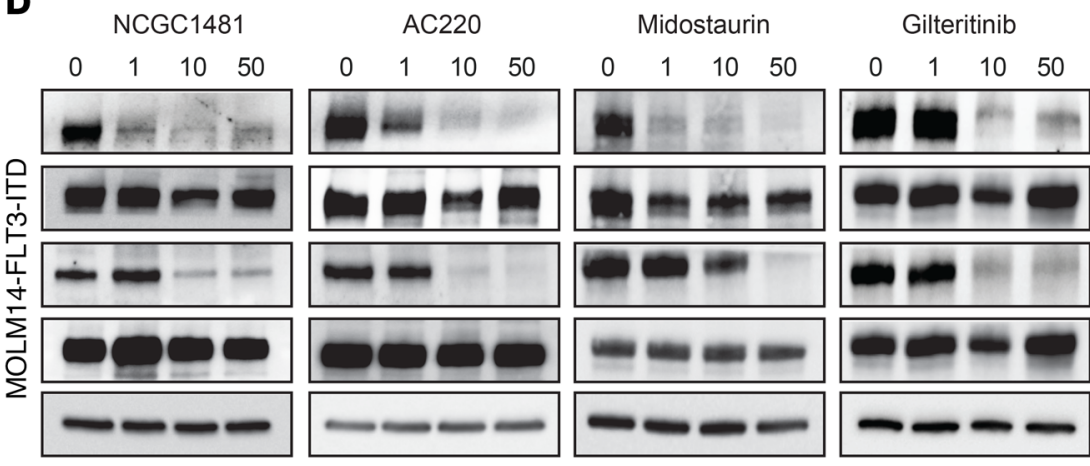

Crenolanib

$\mathbf{E}$
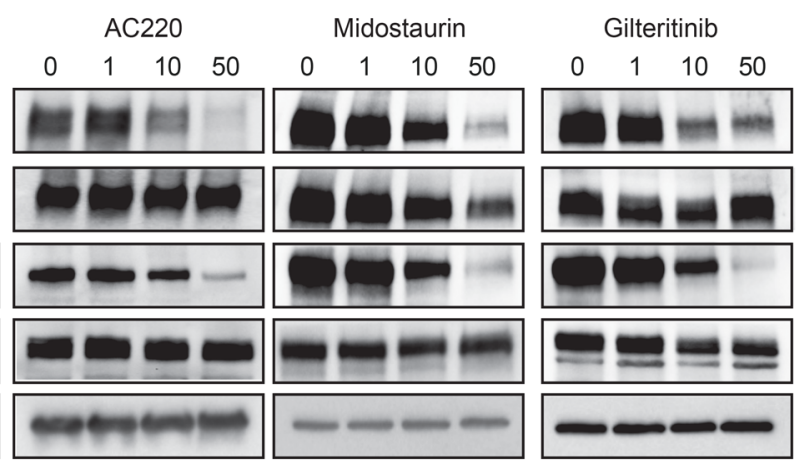

Crenolanib

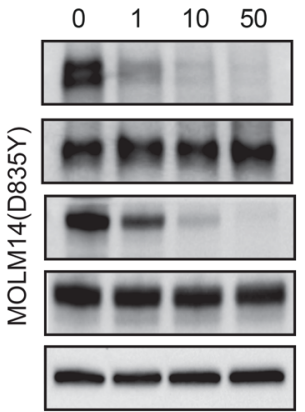

Figure 1. Chemical structure and inhibitory function of NCGC1481. (A) Chemical structure of NCGC1481. (B) A ribbon/surface representation of the cocrystal of NCGC1481-FLT3 (PDB: 6IL3) and a ribbon/surface representation of the cocrystal of AC220-FLT3 (PDB: 4XUF). (C) Detailed view of the Asp835-Ser838 hydrogen bond formed in the cocrystal of NCGC1481-FLT3 relative to loss of this hydrogen bond in the cocrystal of AC220-FLT3 (left 2 panels). Detailed view of the F691-ligand hydrogen bond formed in the cocrystal of NCGC1481-FLT3 and the cocrystal of AC220-FLT3 (right 2 panels). (D) Immunoblotting of isogenic MOLM14-FLT3-ITD cell lines treated with the indicated inhibitors for 90 minutes. (E) Immmunoblotting of MOLM14-FLT3-ITD(D835Y) cell lines treated with the indicated inhibitors for 90 minutes. See complete unedited blots in the supplemental material. 

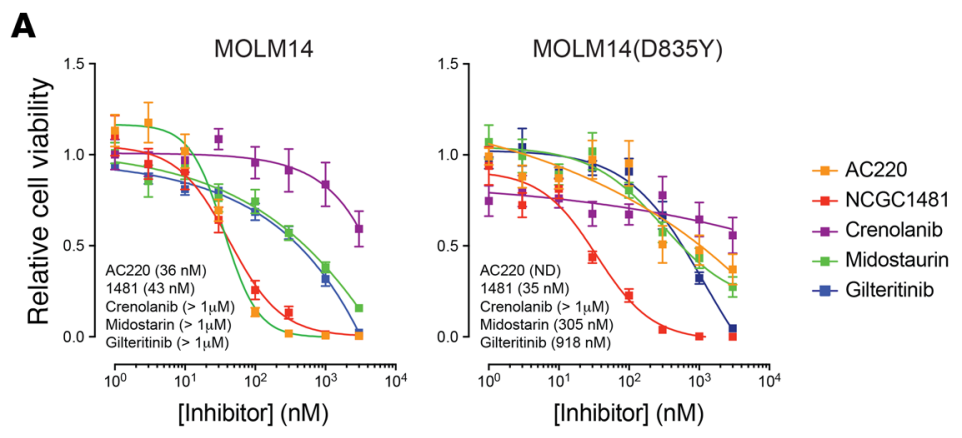

\section{B}

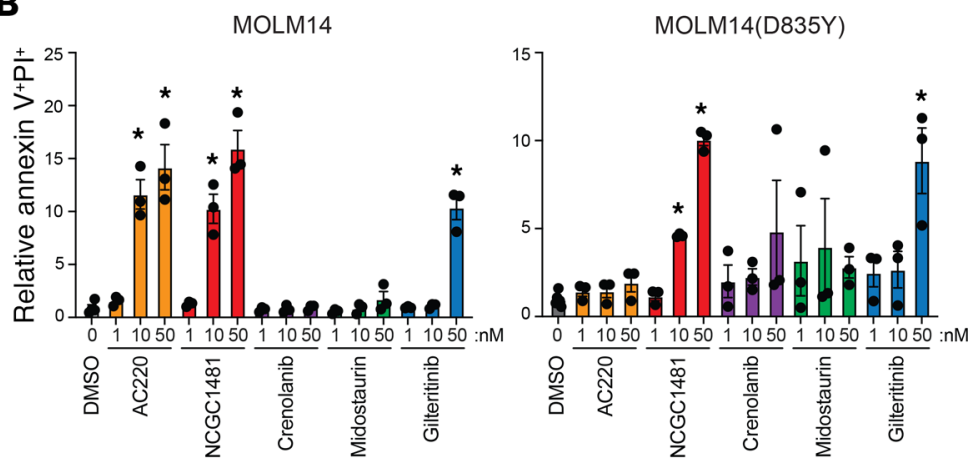

C
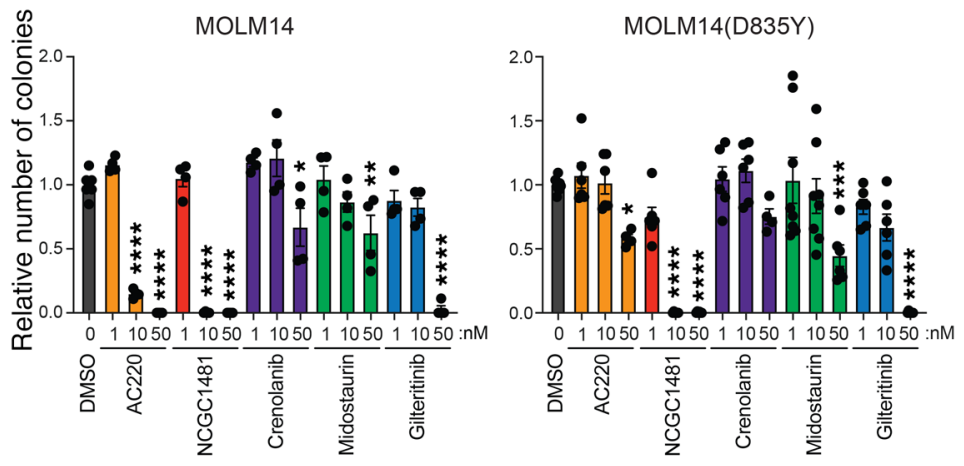

D

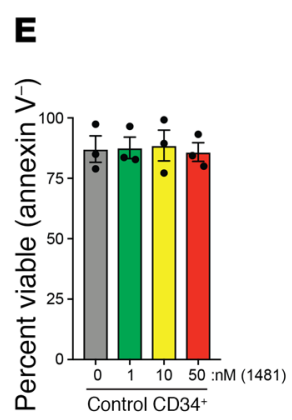

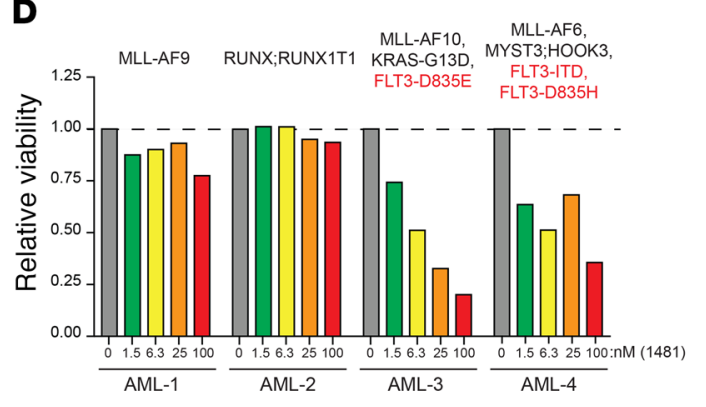

Figure 2. NCGC1481 targets clinically relevant FLT3-mutant AML cells in vitro. (A) Viability of MOLM14-FLT3-ITD and MOLM14-FLT3-ITD(D835Y) cells treated with the indicated inhibitors for 72 hours. Values are expressed as the mean \pm SEM of 6-12 independent samples. (B) Annexin $V$ and propidium iodide (PI) staining of MOLM14-FLT3-ITD and MOLM14-FLT3ITD(D835Y) cells treated with the indicated inhibitors for 48 hours. Values are expressed as the mean \pm SEM of biological triplicate samples. ${ }^{*} P<0.05$, by Dunnett's multiple corrections test. (C) Colony formation of MOLM14-FLT3-ITD and MOLM14FLT3-ITD(D835Y) cells treated with the indicated inhibitors. Colony formation was determined after 7 days. Values are expressed as the mean \pm SD of $4-10$ independent samples. ${ }^{*} P$ $<0.05$, ${ }^{* *} P<0.005$, ${ }^{* *} P<0.0005$, and ${ }^{* * *} P<0.0001$, by Dunnett's multiple corrections test. (D) Viability of primary AML cells was determined in the presence of NCGC1481 for 48 hours by trypan blue exclusion. The mutational status of the patients' cells is indicated above. (E) Viability of cord blood-derived CD34+ cells from 3 donors was determined in the presence of NCGC1481 for 48 hours by annexin V and PI staining. Data represent the mean \pm SEM

tration values are not identical to ones previously reported (17-20), which could be accounted for by variations in experimental conditions, the relative activities of the FLT3 inhibitors across assays and FLT3-mutant cell lines remained consistent (Figure 2A and Supplemental Figure 1). Compared with the FLT3-ITD(D835Y) cell line, only NCGC1481 was found to possess activity below $100 \mathrm{nM}$ $\left(\mathrm{IC}_{50}=35 \mathrm{nM}\right)$ (Figure 2A). The lack of activity for AC220 and midostaurin is consistent with clinical data showing that resistance to FLT3i results from acquired and/or subclonal FLT3 mutants $(7,11,12)$. We found that the reduced viability of FLT3-ITD and FLT3-ITD(D835Y) MOLM14 cells in the presence of NCGC1481 was due to increased apoptosis (Figure 2B).

A more rigorous assessment of antileukemic activity was made by measuring the clonogenic potential of MOLM14 cells exposed to NCGC1481 in methylcellulose assays. We found that NCGC1481 significantly suppressed leukemic colony formation of MOLM14 cells harboring FLT3-ITD and FLT3-ITD(D835Y) at $10 \mathrm{nM}$ (Figure 2C). Gilteritinib suppressed leukemic colony formation at $50 \mathrm{nM}$ in both cell lines, whereas AC220 was only effective against FLT3-ITD MOLM14 cells at this concentration. Midostaurin and crenolanib did not significantly inhibit leukemic colony formation of either FLT3-ITD or FLT3-ITD(D835Y) MOLM14 cells at concentrations up to $50 \mathrm{nM}$. Given the clinenvironment plays a critical role in determining enzyme conformation, activity, and susceptibility to ligand binding and inhibition.

To examine the cell-killing potential of NCGC1481 relative to that of the other drugs profiled in this study, we examined the viability of both isogenic AML cell lines harboring FLT3-ITD and FLT3ITD(D835Y), following drug exposure using a luminescence-based viability assay. Both AC220 and NCGC1481 had a strong cell-killing phenotype $\left(\mathrm{IC}_{50}=36 \mathrm{nM}\right.$ and $43 \mathrm{nM}$, respectively), whereas gilteritinib, midostaurin, and crenolanib exerted more modest cytotoxicity $\left(\mathrm{IC}_{50}>900 \mathrm{nM}\right)$ (Figure 2A). Although these inhibitory concenical ramifications of the F691 mutation, we examined the efficacy of NCGC1481 against the F691L FLT3 mutant. Consistent with its binding potential, NCGC1481 effectively suppressed FLT3-ITD(F691L) MOLM14 cells as demonstrated by reduced phosphorylated FLT3 (p-FLT3) and p-STAT5 (Supplemental Figure 2A). Gilteritinib effectively suppressed FLT3 signaling in FLT3-ITD(F691L) MOLM14 cells; however, it was less effective at suppressing the leukemic function of MOLM14(F691L) cells relative to NCGC1481 (Supplemental Figure 2, A and B). Importantly, the activity of NCGC1481 and the relative activity of key 
A

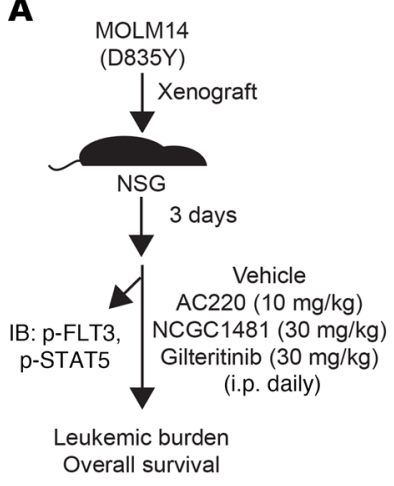

D

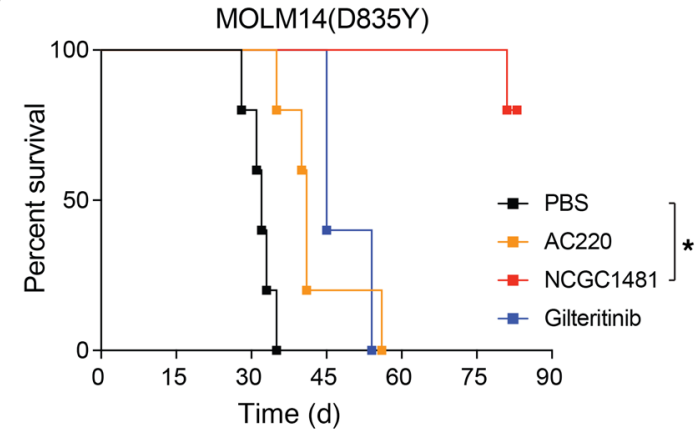

B

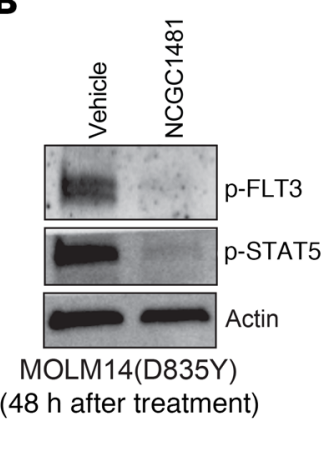

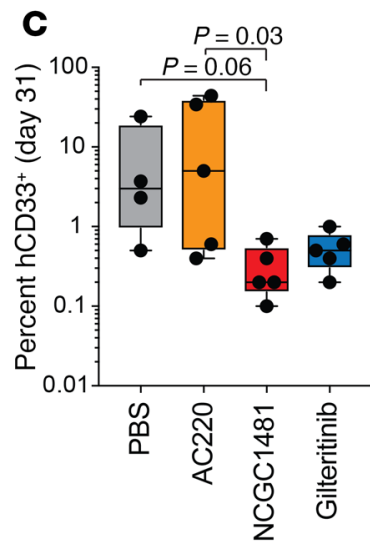

$\mathbf{E}$

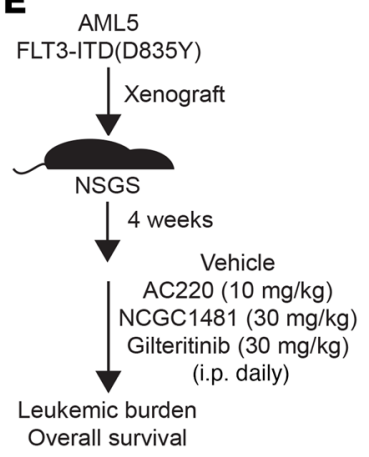

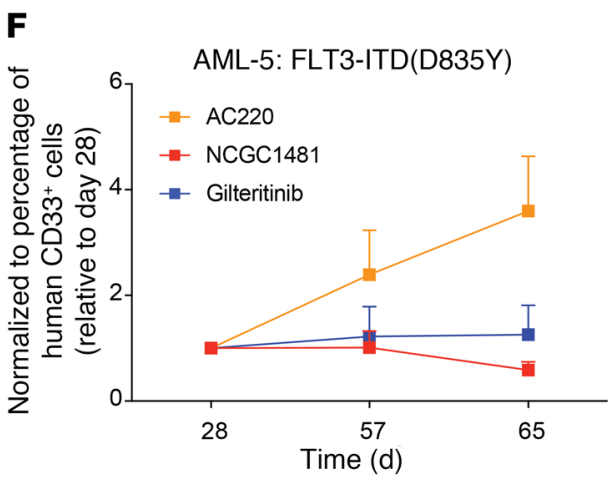

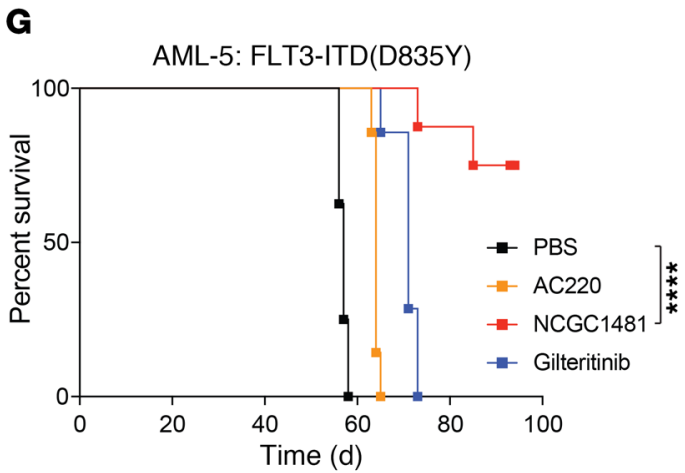

Figure 3. NCGC1481 targets clinically relevant FLT3-mutant AML cells in vivo. (A) Experimental design of the xenograft studies. (B) After treatment with NCGC1481, MOLM14-FLT3-ITD(D835Y) cells (human CD33 ${ }^{+}$hCD33 $]$) were isolated from the BM for immunoblot analysis. (C) BM aspirates were analyzed for leukemic burden on day 31 after transplantation ( $n=5$ mice per condition). Values are expressed as the mean \pm SEM for individual mice. $P=0.06$ for PBS versus NCGC1481; $P=0.03$ for AC220 versus NCGC1481; Dunn's multiple comparisons test. (D) Leukemia-free survival of NSG mice xenografted with MOLM14-FLT3-ITD(D835Y) cells and treated with the indicated FLT3i or vehicle ( $n=5$ mice per group). ${ }^{*} P<0.05$, by log-rank (Mantel-Cox) test. (E) Experimental design of patient-derived xenograft studies. (F) BM was analyzed for leukemic burden around day 65 after transplantation ( $n=5-7$ mice per condition). Values are expressed relative to baseline values on day 28 for individual mice. (C) Leukemia-free survival of NSGS mice xenografted with AML-5 cells and treated with the indicated FLT3i or vehicle ( $n=7-8$ mice per group). ${ }^{* * *} P<0.0001$, by log-rank (Mantel-Cox) test.

agents (e.g., AC220 and gilteritinib) were consistent across multiple FLT3-mutant AML cell models, culture formats, and assay conditions (Supplemental Figures 2 and 3).

We next evaluated the selectivity and potency of NCGC1481 on patient-derived AML samples and healthy hematopoietic cells (Supplemental Table 2). In a panel of 4 primary AML patients' samples, NCGC1481 was equally effective against FLT3(D835E) (AML-3) and FLT3-ITD;FLT3(D835Y) (AML-4) mutant AML cells, whereas it did not exhibit antileukemic activity against 2 of the AML samples (AML-1 and AML-2) without FLT3 mutations (Figure 2D). NCGC1481 did not affect the viability (Figure 2E) or colony formation of healthy $\mathrm{CD} 34^{+}$cells at similar concentrations (Supplemental Figure 4). These findings indicate that NCGC1481 is exquisitely potent at suppressing FLT3 signaling and leukemic cell function of clinically relevant mutants in vitro.

It was critical to assess whether the superior inhibitory activity of NCGC1481 extended to the most common clinically relevant FLT3 ITD(D835Y) mutant in vivo. As such, we established MOLM14(D835Y) tumors in NOD-SCID IL2R $\gamma^{-/}$(NSG) mice and evaluated the activity of NCGC1481 (30 mg/kg per day) (Figure 3A). At this dose, NCGC1481 had acceptable pharmacokinetic properties in mice, possessed no adverse hematologic 
toxicity, and elicited no significant weight changes over 6 weeks of continuous dosing (Supplemental Figure 5). To gain a comparative assessment for key agents, we included AC220 and gilteritinib (dosed at $10 \mathrm{mg} / \mathrm{kg}$ and $30 \mathrm{mg} / \mathrm{kg}$, respectively, on the basis of previous studies [refs. 6, 21]). Importantly, we confirmed that phosphorylation of both FLT3 and STAT5 was substantially reduced in MOLM14(D835Y) cells isolated from mice treated with NCGC1481 compared with mice given vehicle (Figure 3B). On day 30 of treatment, NCGC1481 conferred a 96\% reduction in leukemic burden in the bone marrow (BM) compared with mice receiving vehicle, and a $98 \%$ and $41 \%$ reduction relative to AC220- or gilteritinib-treated mice, respectively (Figure 3C). At the same dose, NCGC1481 administration significantly extended the overall median survival of mice xenografted with MOLM14(D835Y) cells $(P=0.0018)$ (Figure 3D). Most of the mice treated with NCGC1481 did not die from leukemia beyond 80 days of treatment (at which point the experiment was terminated) (Figure 3D), compared with the survival of mice treated with vehicle (median survival $=32$ days), AC220 (median survival $=41$ days), or gilteritinib (median survival $=45$ days).

We next evaluated the effects of NCGC1481 in patientderived xenografts. We administered NCGC1481 (30 mg/kg per day) to NOD/LtSz-SCID IL2RG-SGM3 (NSGS) mice xenografted with patient-derived AML cells harboring FLT3-ITD(D835Y) (AML-5) (Figure 3E). NCGC1481 significantly extended the overall survival of mice xenografted with patient-derived FLT3-ITD(D835Y) AML cells $(P<0.0001)$. By day 65 after transplantation, the xenografts treated with NCGC1481 had a stable leukemic burden relative to the baseline quantification of leukemic burden on day 28. Notably, NCGC1481-treated mice had a more stable leukemic burden than did AC220- or gilteritinib-treated mice on day 65, when all of the AC220-treated mice were moribund (Figure $3 \mathrm{~F}$ and Supplemental Figure 6). Compared with mice treated with vehicle (median survival $=57$ days), AC220 (median survival $=64$ days), or gilteritinib (median survival $=71$ days), 6 of 8 mice in the NCGC1481-treated group were alive on day 93, when the treatment was discontinued (Figure 3G). Taken together, these data show that NCGC1481 is a potent and efficacious small-molecule inhibitor of FLT3-ITD and clinically relevant mutants, with superior inhibitory activity compared with the current generation of FLT3 inhibitors.

In summary, we have identified a small-molecule inhibitor of clinically relevant FLT3 mutants that exploits a unique binding pose that is unaffected by common active site mutations in FLT3 and, consequently, retains remarkable cytotoxic potential versus FLT3-mutant AML cells harboring these mutations both in vitro and in vivo. We recently reported that NCGC1481 was also effective at overcoming adaptive resistance to FLT3 inhibitors in AML by targeting immune signaling pathways via direct inhibition of IL-1 receptor-associated kinase 1 (IRAK1) and IRAK4 (15). Collectively, these data support the possibility that NCGC1481 can overcome both target-dependent and target-independent resistance mechanisms and has the potential to improve the outcome of patients with relapsed and refractory FLT3-mutant AML.

\section{Methods}

Detailed information regarding the materials, methods, and statistical analyses used in this study can be found in the Supplemental Methods.

Study approval. Human $\mathrm{CD}^{+} 4^{+}$umbilical cord blood and BM cells were obtained from the Translational Research Development Support Laboratory of Cincinnati Children's Hospital under a protocol approved by this institute. PRoXe samples were obtained within the framework of routine diagnostic BM aspirations after written informed consent was obtained, in accordance with the Declaration of Helsinki. All mice were bred, housed, and handled in the Association for Assessment and Accreditation of Laboratory Animal Careaccredited animal facility of Cincinnati Children's Hospital Medical Center. All animal procedures were performed in accordance with the protocol approved by the Institutional Animal Care and Use Committee at Cincinnati Children's Hospital (IACUC2019-0072).

\section{Author contributions}

LMJ, KM, LB, KH, MMW, JKJ, KMW, JS, FJ, XZ, PS, AW, MW, and EOB performed experiments and analyzed data. JS and FJ performed protein-ligand crystallization studies. LMJ, KM, LB, XX, GJT, SBH, MW, EOB, JPP, DTS, and CJT analyzed data. LMJ, DTS, and CJT wrote the manuscript. All authors edited the manuscript. DTS and CJT conceived the project and managed all studies.

\section{Acknowledgments}

This work was supported by the Cincinnati Children's Hospital Research Foundation, the Leukemia Lymphoma Society, CancerFree KIDS, the NIH (R35HL135787 and RO1DK113639, to DTS), and by intramural research programs of the National Center for Advancing Translational Sciences and the NCI. LMJ is supported by a St. Baldrick's Foundation Research Fellowship (ID: 524748). KM is supported by a NIH Research Training and Career Development Grant (F31CA217140). MW is supported by a NCI grant (R50CA211404). DTS is a Leukemia Lymphoma Society Scholar. We thank Jeff Bailey and Victoria Summey of the Comprehensive Mouse and Cancer Core (Cincinnati Children's Hospital Medical Center) for their assistance.

Address correspondence to: Daniel T. Starczynowski, Division of Experimental Hematology and Cancer Biology, Cincinnati Children's Hospital Medical Center, 3333 Burnet Avenue, Cincinnati, Ohio 45229, USA. Phone: 513.803.5317; Email: daniel. starczynowski@cchmc.org. Or to: Craig J. Thomas, Division of Preclinical Innovation, NIH Chemical Genomics Center, National Center for Advancing Translational Sciences, 9800 Medical Center Drive, Rockville, Maryland 20892, USA. Phone: 301.827.1798; Email: craigt@mail.nih.gov.
1. Levis M, Small D. FLT3: ITDoes matter in leukemia. Leukemia. 2003;17(9):1738-1752.

2. Pratz KW, Levis M. How I treat FLT3-mutated AML. Blood. 2017;129(5):565-571.

3. Larrosa-Garcia M, Baer MR. FLT3 Inhibitors in acute myeloid leukemia: current status and future directions. Mol Cancer Ther. 2017;16(6):991-1001.

4. Levis M. Midostaurin approved for FLT3-mutated AML. Blood. 2017;129(26):3403-3406.
5. Sidaway P. Quizartinib tested in patients with high-risk AML. Nat Rev Clin Oncol. 2018;15(9):532.

6. Mori M, et al. Gilteritinib, a FLT3/AXL inhibitor, shows antileukemic activity in mouse models of 
FLT3 mutated acute myeloid leukemia. Invest New Drugs. 2017;35(5):556-565.

7. Daver N, et al. Secondary mutations as mediators of resistance to targeted therapy in leukemia. Blood. 2015;125(21):3236-3245.

8. McMahon CM, et al. Mechanisms of acquired resistance to gilteritinib therapy in relapsed and refractory FLT3-mutated acute myeloid leukemia. Blood. 2017;130(Suppl 1):295.

9. Perl AE, et al. Selective inhibition of FLT3 by gilteritinib in relapsed or refractory acute myeloid leukaemia: a multicentre, first-in-human, open-label, phase 1-2 study. Lancet Oncol. 2017;18(8):1061-1075.

10. Piloto O, Wright M, Brown P, Kim KT, Levis M, Small D. Prolonged exposure to FLT3 inhibitors leads to resistance via activation of parallel signaling pathways. Blood. 2007;109(4):1643-1652.

11. Smith CC, et al. Heterogeneous resistance to quizartinib in acute myeloid leukemia revealed by single-cell analysis. Blood. 2017;130(1):48-58.
12. Smith CC, et al. Validation of ITD mutations in FLT3 as a therapeutic target in human acute myeloid leukaemia. Nature. 2012;485(7397):260-263.

13. Smith CC, Lin K, Stecula A, Sali A, Shah NP. FLT3 D835 mutations confer differential resistance to type II FLT3 inhibitors. Leukemia. 2015;29(12):2390-2392.

14. Vijayan RS, et al. Conformational analysis of the DFG-out kinase motif and biochemical profiling of structurally validated type II inhibitors. JMed Chem. 2015;58(1):466-479.

15. Melgar K, et al. Overcoming adaptive therapy resistance in AML by targeting immune response pathways. Sci Transl Med. 2019;11(508):eaaw8828.

16. Smith CC, et al. Characterizing and overriding the structural mechanism of the quizartinibresistant FLT3 "gatekeeper" F691L mutation with PLX3397. Cancer Discov. 2015;5(6):668-679.

17. Furukawa Y, et al. Divergent cytotoxic effects of PKC412 in combination with conventional antileukemic agents in FLT3 mutation-positive versus -negative leukemia cell lines. Leukemia. 2007;21(5):1005-1014.

18. Kampa-Schittenhelm KM, Heinrich MC, Akmut F, Döhner H, Döhner K, Schittenhelm MM. Quizartinib (AC220) is a potent second generation class III tyrosine kinase inhibitor that displays a distinct inhibition profile against mutant-FLT3, -PDGFRA and -KIT isoforms. $\mathrm{Mol}$ Cancer. 2013;12:19.

19. Lee LY, et al. Preclinical studies of gilteritinib, a next-generation FLT3 inhibitor. Blood. 2017;129(2):257-260.

20. Smith CC, et al. Crenolanib is a selective type I pan-FLT3 inhibitor. Proc Natl Acad Sci US A. 2014;111(14):5319-5324.

21. Zarrinkar PP, et al. AC220 is a uniquely potent and selective inhibitor of FLT3 for the treatment of acute myeloid leukemia (AML). Blood. 2009;114(14):2984-2992. 